\title{
The McCollough effect: Influence of several kinds of visual stimulation on decay rate
}

\author{
D. SKOWBO, T. GENTRY, B. TIMNEY, and R. B. MORANT \\ Brandeis University, Waltham, Massachusetts 02154
}

\begin{abstract}
An experiment was conducted to determine whether the decay rate of the McCollough effect could be differentially influenced by the type of visual stimulation that followed its induction. After acquiring this effect, Os were exposed to achromatic gratings, homogeneous chromatic fields, natural visual stimulation, or complete darkness. Exposure to achromatic gratings caused a marked fading of the effect; the other types of stimulation were associated with similar and much less rapid decay.
\end{abstract}

In 1965, McCollough discovered a new color aftereffect that had an exceptionally long duration. She exposed Ss to a grating of vertical black stripes on an orange background which alternated every few seconds with a horizontal grating on a blue background. After several minutes of exposure, Ss presented with horizontal and vertical black and white "test" gratings reported that the patterns appeared tinted with the hue complementary to that which had been paired with each orientation during adaptation.

Since McCollough's report (1965), it has been established that the color of the McCollough effect (ME) is dependent on spatial characteristics of the grating patterns used for both induction of the effect and testing for its presence; for example, in order for the $\mathrm{ME}$ to appear, test patterns must be close in orientation and spatial frequency to the patterns used for adaptation (Teft \& Clark, 1968). This dependence might reflect some aspect of the way in which color and contour are processed neurophysiologically, and there have been single-cell-level models formulated which describe the ME in terms of "fatigued" units (e.g., Murch, 1972a). However, the persistence of this effect-it has been estimated to last days or even weeks (Stromeyer \& Mansfield, 1970; Stromeyer, 1971)-is difficult to explain within a simple neurophysiological framework.

It has been remarked that aftereffects like the ME have characteristics which suggest a perceptual learning mechanism (Mayhew \& Anstis, 1972). We approached the question of whether this effect might be maintained over long periods as a learned response by asking how its rate of decay could be influenced by the type of visual stimulation following its induction. For example, a learned response should be retained in the absence of stimulation (complete darkness), whereas fatigued units might be expected to recover relatively quickly under such a condition.

\section{METHODS}

Our basic procedure was to adapt each of three trained Os to a $M E$ adaptation sequence and then expose them to one of several types of postadaptation stimulation (PAS). The ME was induced by alternately viewing a vertical and a horizontal grating ( 5 cycles/deg) projected on either a green (Wratten No. 53) or violet (Wratten No. 34A) background. The two gratings were alter nated every $5 \mathrm{sec}$ for a $10-\mathrm{min}$ period. The filter/orientation combinations were changed from session to session for each $O$. Each ME adaptation sequence was followed by $50 \mathrm{~min}$ of exposure to one of the following PAS conditions: (1) alternating vertical and horizontal achromatic gratings; (2) alternating green and violet homogeneous fields; (3) "natural" visual stimulation (the $\mathrm{O}$ walked about freely in the building and out of doors); (4) complete darkness; (5) alternating chromatic gratings having the filter/orientation pairing opposite to that used to induce the ME-a configuration which has been found to "cancel" MEs (Stromeyer, 1969). In PAS Conditions 1, 2, and 5, the rate at which the fields were alternated, their hue, and the spatial frequency of the gratings were those specified for $\mathrm{ME}$ adaptation. In Experiment 1, the PAS period was divided into five 10-min segments; in Experiment 2, exposure was continuous for $50 \mathrm{~min}$, and PAS Condition 5 was omitted. ME adaptation and PAS sequences were always presented monocularly, to the left eye; during these periods, the right eye was covered. Each $\mathrm{O}$ was run on each condition, with at least $24 \mathrm{~h}$ elapsing between sessions. At the end of each session, any remaining effects were neutralized by brief exposures to the filter/orientation combination opposite to that used in the ME adaptation or, in the case of PAS Condition 5, to the opposite of the PAS combination.

To evaluate the influence of the PAS conditions on the rate of decay of the $\mathrm{ME}$, it was necessary to obtain quantifiable measures of the ME. These were obtained with a specially designed tristimulus projection colorimeter. ${ }^{1}$ With this instrument, a homogeneous chromatic field seen with the right eye could be adjusted to match the ME seen on test gratings viewed with the left; color matches could then be converted into CIE $x, y$ coordinates. The matches were made before and after ME adaptation in both experiments, after each of the five PAS segments in the first experiment, and af ter the entire PAS period in the second. Prior to each matching block, $7 \mathrm{~min}$ (Experiment 1) or $12 \mathrm{~min}$ (Experiment 2) of binocular adaptation to homogeneous fields was provided to insure equivalent levels of light adaptation in the two eyes and to allow any successive contrast effects of chromatic stimulation to fade.

\section{RESULTS}

For each session, the magnitude of the ME was defined as the distance in CIE color space between matches made before ME adaptation and those made after either the adaptation or the PAS period(s). The 

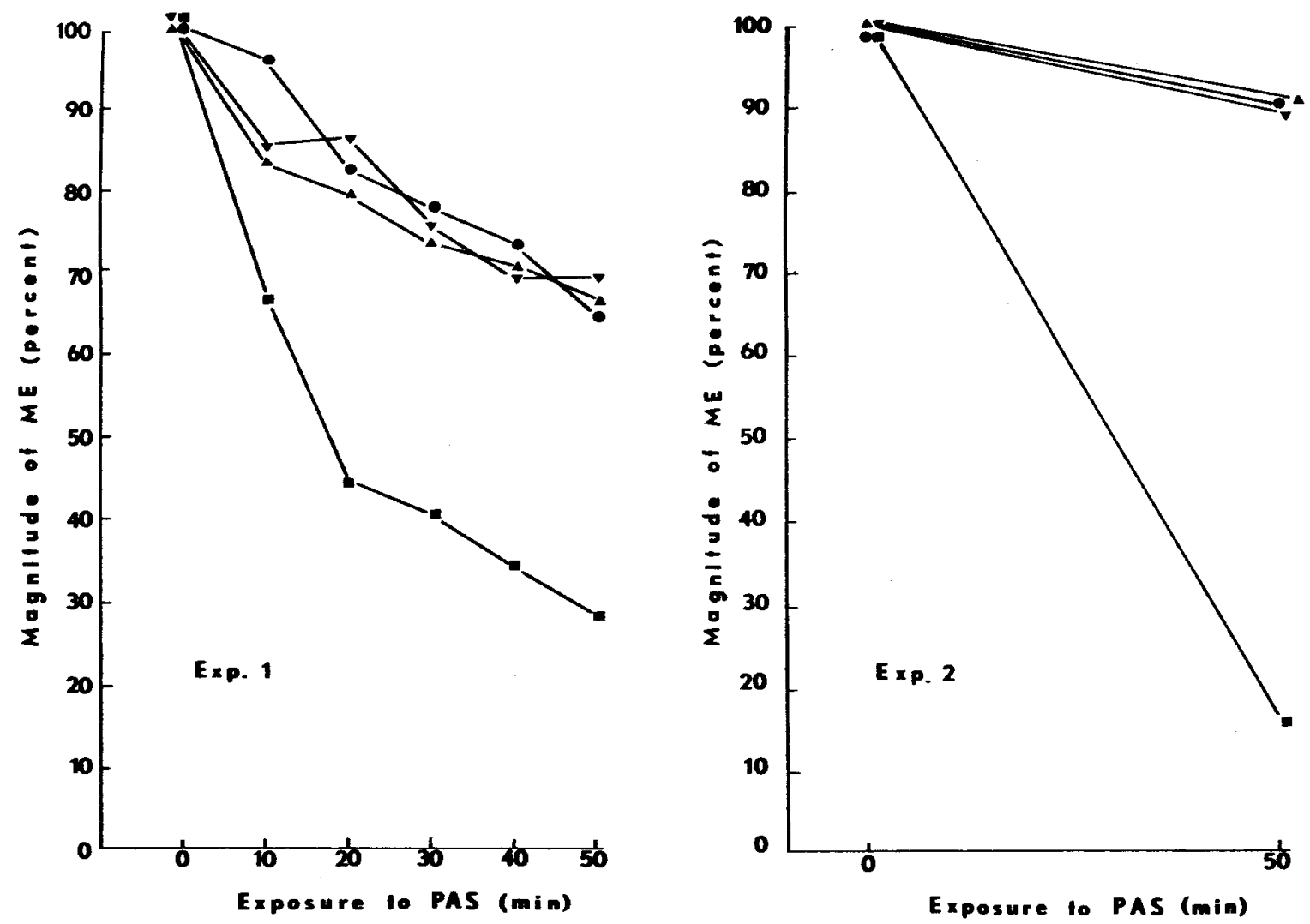

Figs. 1 and 2. Influence of PAS conditions on decay of the ME. The ordinate is the percentage of the ME measured before PAS which remains after PAS. The abscissa is the amount of time of exposure to PAS. In Experiment 1 , there were five 10-min segments of PAS; in Experiment 2, PAS was continuous for $50 \mathrm{~min}$. The PAS conditions are represented by the

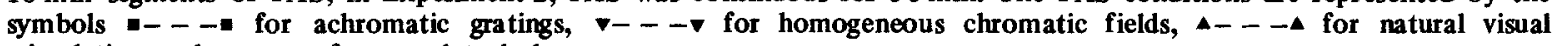
stimulation, and $\bullet--\rightarrow$ for complete darkness.

data were normalized so that the initial ME (the difference between pre- and post-ME adaptation matches, prior to any PAS) could be expressed as $100 \%$, and matches made after any PAS as fractions of this value.

Since the data for all three Os for both the vertical and horizontal gratings showed essentially the same patterns of decay, the results were averaged. The results of Experiment 1 are presented in Fig. 1, those of Experiment 2, in Fig. 2.

A plot of PAS Condition 5 is omitted from Fig. 1. For all Os, matches made after the first 10-min block of this stimulation indicated that not only had the original ME been cancelled, but that a slight "reversed" effect had been built up-in other words, the magnitude of the ME after this exposure block would have fallen below $0 \%$. Matches made following subsequent exposure periods in this condition showed a continuing buildup of this opposite effect.

Of the other PAS conditions, in both experiments, postadaptation exposure to achromatic gratings caused the most rapid decayof the ME. The remaining three conditions were associated with a less rapid decline in magnitude of the ME and were indistinguishable from one another.

\section{DISCUSSION}

In evaluating our findings, we have considered the possibility that, given the presence of the ME, subsequent viewing of achromatic gratings might be like viewing a low-saturation neutralization configuration. Thus, exposure to the former type of stimulation might weaken the effect because of its perceptual resemblance to the latter-a pattern which was found in our experiment to erase MEs quickly. A point which argues against this analogy is that, according to Os, the PAS gratings appeared to be quite achromatic: the ME was not visible on them nearly to the extent that it showed up on the test patterns. There may be two reasons for this. First, MEs often are not seen well unless the spatial stimuli on which they depend are presented side by side. In the PAS condition, the horizontal and vertical gratings were alternated. Secondly, the PAS gratings were slightly brighter than the test gratings: we have observed in other experiments that MEs are seen best on 
rather dim test patterns, and that they can disappear altogether if the luminance is increased by only a few foot-lamberts.

Neurophysiological models that have been proposed to account for the ME have invoked a chromatic adaptation process-in terms of edge detectors which can be selectively color-adapted (McCollough, 1965), or adaptation in color- and orientation-specific channels (Held \& Shattuck, 1971), or fatigue of color receptors which feed into contour-detecting units (Murch, 1972a). On the basis of any of these models, it would be expected that periods of darkness would facilitate recovery from adaptation effects. Moreover, exposure to achromatic gratings should not have had any influence on effects resulting from chromatic adaptation of edge detectors or color receptors, nor should it have affected adaptation in channels selective for color and orientation. Our observations, therefore, are inconsistent with predictions based on any of these models. In addition, neural adaptation models seem unable to account for the long duration of the ME, since neural fatigue is usually not thought to persist for days or weeks.

Postulation of a learning process which is based upon chromatic adaptation could, however, account for the results we obtained, as well as for the duration of the $\mathrm{ME}$. If the aftereffect of color is considered as a conditioned response, it is possible that line orientation (or the firing of units tuned to a particular orientation) acts as a conditioned stimulus which becomes associated with adaptation in color units. The rapid decay of the ME which accompanies exposure to achromatic gratings could, according to this line of reasoning, be described as the extinction of a learned response. Most other kinds of stimulation would not be expected to interfere with the association which had been built up between color and orientation; however, a neutralization configuration-essentially a reversal in stimulus contiguities-could quickly abolish the response.

\section{REFERENCES}

Held, R., \& Shattuck, S. Color and edge-sensitive channels in the human visual system: Tuning for orientation. Science, 1971 , $174,314-316$.

Mayhew, J., \& Anstis, S. Movement aftereffects contingent on color, intensity, and pattern. Perception \& Psychophysics, $1972,12,77-85$

McCollough, C. Color adaptation of edge detectors in the human visual sy stem. Science, 1965, 149, $1115-1116$.

Murch, G. Binocular relationships in a size and color orientation specific aftereffect. Journal of Experimental Psychology, $1972 a, 93,30-34$.

Murch $G$. CIE $x, y$ coordinates from an inexpensive projection colorimeter. Behavior Research Methods \& Instrumentation, $1972 \mathrm{~b}, 4,3-5$.

Riggs, L. A. A projection colorimeter. American Journal of Psychology, 1964, 77, 129-134.

Stromeyer C. Further studies of the McCollough effect. Perception \& Psychophysics, 1969, 6, 105-110.

Stromeyer, C. MeCollough effect analogs of two-color projections. Vision Research, 1971, 11, 969-978.

Stromeyer, C., \& Mansfield, $\dot{R}$. Colored aftereffects produced with moving edges. Perception \& Psychophysics, 1970, 7, 108-114.

Teft, L.. \& Clark, F. The effects of stimulus density on orientation specific aftereffects of color adaptation. Psychonomic Science, 1968, 11, 265-266.

\section{NOTE}

1. This instrument was modeled after a design by Riggs (1964); we thank Billy $R$. Wooten for his assistance in its construction and calibration. Our choice of primaries required calibration procedures other than those suggested by Murch (1972b).

(Received for publication October 22, 1973; revision received February 2,1974 ) 